\title{
Ischemic Stroke. Use of the Keypad for Neurophysiological Rehabilitation in the Sickwhen Stroke and Cerebelum Stroke. Opis in Public Neurophysiological Rehabilitation during the Training of Physicians and Rehabilitators at the Centre of the Key-Source, Molecular Method Biocybernethycz (Non-Invasive), Patient Relationship, 2007 Year
}

\author{
Patient* \\ Infectious Diseases Specialist, Centrum Klawitherapy -Sourcelo, Strzeniówka, Ul. Blackberries, Nadarzyn, Warsaw, Poland \\ *Corresponding Author: Patient, Infectious Diseases Specialist, Centrum Klawitherapy -Sourcelo, Strzeniówka, Ul. Blackberries, \\ Nadarzyn, Warsaw, Poland.
}

Received: September 03, 2019; Published: October 11, 2019

DOI: $10.31080 /$ ASMS.2019.03.0435

\begin{abstract}
Key therapy is a new diagnostic and therapeutic direction in medicine that began in the years 60 TO 20th century. Creator of this new, Pioneery method is the Polish psychologist Dr. Ferdinand Barbasiewicz.

The mechanism of action of the-Key and the scientific basis. To Use this method in the diagnosis and therapy It is necessary to have a detailed knowledge: Neurology, anatomy including primarily Nervous system and human circulatory systems. The scientific basis for this method differs from acupuncture and acupressure. The diagnostic-Therapeutic method Klawi therapy is non-invasive and so far there has been no contraindications for its use. The following Are exceptions: severe malnutrition and patient after organ transplantation. From Experience Creator Kthat this method is extremely effective in the treatment of M. In.in the treatment of diseases of the nervous system with particular emphasis on Shard and disseminated it, hardened and Lateralitatroticit, strike breaking. Keywords: Keyboard Therapy; Acupuncture; Biocybernetics; Stroke
\end{abstract}

\section{Patient and Method}

The key therapy was used in 51-year-old patients after stroke and cerebelate.

\section{Case description}

51-year-old Men on August 2, 2008 in the evening suffered suddenly exacerbating dizziness occurring with vomiting and loss of consciousness. At the same time, the right limb and lower limbs have progressed. After about 40 minutes the sick was admitted to the SOR branch. After the initial analysis, the patient was placed in an impact ward. In a medical examination in the sick, I found no m.in. urinary and fecal retention; In the neurological study in the detachment, Demetris's was found in cerebeltic trials on the right side. In the study, the magnetic resonance of the head was shown in the white of both hemispheres of the brain around-ventricular and in the carboline on the right side in the vicinity of chamber IV of the vascularly-derived foci of elevated signal in T2 Images- and post-stroke fire in the right hemisphere of the cerebellum. During the first 4 days of the stay in the neurological department the pa- tient was conscious though afterD-sleeper. The patient was taking the following preparations: Metocard, Areplex, Polfilin, Betaserc, Polprazol, Depakine chrono. After 9 days of stay, the sick was tried to plume, and the Solares Litany was startedin the ward. On the 13th Day of stay in the patient 'sZpitalu was checked out to the house in a general condition somewhat better.The patient continued to be lying and required third-party care. From mid-September to mid-December 2008 The patient was rehabilitated in a home environment. In October 2008, the The sick began to walk with the help of a "Balconician", still the stringu required help with daily activities. In the period September-December 2008, the patient had symptoms such as shoulder syndromes, pellin syndromes, increased lumbar pain, Chronic pain ofthe right elbow, which while wearing The extremities on the right will prevent the Any action by the patient. In addition, there were still problems with urination and stool. In December 2008, on the skin of the whole body, the sick noticed the presence of white spots of different sizes - diameter from $1 \mathrm{mmto} 4 \mathrm{~mm}$. Within tyc $\mathrm{H}$, the sensation was lifted. The skin in the sowing parts of the limbs on the right side was dry and rough. A spot of dandruff appeared on the scalp. In the second half 
of December 2008, the The patient performed a control resonance of the head stating a number of minor foci of elevated signal in T2 -dependent images and "flair" in the white essence of both hemispheres of the brain, slightly more than in the study of 05 August 2008. The change located at the top of the right hemisphere of the cerebral is slightly smaller than before. Due to the nature of the changes, the visual potential induced by obtaining the correct image was performed.

Despite taking medications recommended by the caring neurologist, severe dizziness and migraine headaches have become increasingly common in the patient.

On 06.01.2009 R, the sick reported to Dr. Ferdinand Barbarianism in order to carry out the keys.

Description of the sequence of the key treatment used in the patient

1. The course and sequence of treatments of the seven-day treatment by method and Indication therapy stimulatory effect for 6 minutes on therapeutic zones of the cortical and central brain, carboline and spinal cord, in TZW. Destresing from aftershocks.

2. Stimulating effect for 20 minutes on therapeutic zones strengthening the dynamics of the muscle of the blood vessels.

3. Stimulatory effect for 10 minutes on the therapeutic zones unlocking the nervous system sympathetic NAC blood Brain, Cerebellum and the spinal cord.

4. Stimulation effect for 12 minutes on the zones and the centre-and-motor, brain, cerebellum and spinal cord.

5. Stimulatory effect for 60 minutes, abhorting in the surrounding spine the physiology of all the infested peripheral nerve fibers.

6. Stimulation effect for 15 minutes on the algostic and algodystrophatic spots, regulating the neural potentials of their own and inducing the loss of conduction of nerve signals in nerve fibers.

7. Action Cerebrative Other side of the keys for 10 minutes on the Plaka (Fr. Plaque) Demyelinating diagnosed MRI in the brain and cerebelate.

The course and sequence of treatments for the seven-day treatment method and the indication of the key

1. Stimulatory effect for 6 minutes on therapeutic zones of the cortical and central brain, cerebeline and spinal cord.

2. Stimulating effect for 20 minutes on therapeutic zones strengthening the dynamics of vascular muscle blood vessels.

3. Stimulatory effect for 10 minutes on the therapeutic zonesunblocking the nervous system of sympathetic blood vessels of the brain, Cerebeloma
4. and spinal cord.

5. Stimulatory effect for 12 minutes in zones and Cardiac, cerebellitis and prolonged spinal cord; 5) Stimulatory effect for 60 minutes, reaping in the surrounding spine the physiology of all affected peripheral nerve fibers.

6. Stimulatory effects by 15 minutes on the algostic and algodystrophatic spots, regulating the nervous potentials of their own and the awakeningthe conduction of nerve signals in nerve fibers. Cerebration effect the other side of the keys for $10 \mathrm{~min}$ utes on the Plaka (Fr. Plaque) Demyelinating Zdiagn MRI in the brain and cerebelum.

During the first few minutes of the treatment, very light stimulation of the clavicles on the nose, between the eye heads and parallel to the back of the head in the median line under the base of the skull, the patient felt very strong burning (as with burns) and strong difficult to Elimination of the spreading pain. After a few minutes of this baking, the pain disappeared, and in his head felt lightness, relaxation and feeling that he was without discomfort, after about 30 minutes he felt pleasant heat dissipation. Then, when Dr. F. Barbarianism stimulated the clavicles "white" spots (According to his determination of macular algosty- Dead-induced demyelinating neuropathy) appeared in a fraction of a second stabling pain and Smoking as with burns. After a few stimulations on the white spots was wound up with nagging pain and to amazes the sick after about 2 hours regained full efficiency on the right limb of the upper, wrist and toes, completely gave up the pain of theright elbow and sick Mó The voice of the walk without the help of "sphere" because the law of the lower limb became "stronger". The patient was very worried about the recurrence of these ailments. The next day, the treatment was performed again, although the patient was without complaints Dr. Barbarianism explained that subsequent treatments are needed to perception the effect of therapy.

The key treatments were then continued with Dr. F. Barbarianism for another 5 days. After these treatments, they resolved the ailments that annoed the sick from the onset of stroke (sickl. 2008r). The also had changes in the type of fish scales. To this day the sick feels good, there are no complaints.

\section{Discussion}

Human, animal and plant organisms have the ability to selfregulate their functions, self-determination and sometimes selfregulation of lost organs and organ.

Over thousands of years of human existence, many methods have been developed to assist the sick organism in the fight against diseases. Nowadays, such diagnostic and therapeutic methods are widespread, such as: official medicine, homeopathy, Ayurveda, acupuncture, acupressure, herbal remedies, witchcraft, magic.... As you know even officially recognized Homeopathy has not yet been fully clarified. There is no laboratory evidence of how homeopathic medicines work. However, it does not degrade the theoretical assumption of the homeopathic method itself. 
A few thousand years ago in China a therapeutic system was developed to treat a variety of medical conditions through direct effects on the skin. In China it was called "Medicine of Fire and Metal" and in Europe it was only in the 19TH century that the issues of skin-visceral dependence were raised in the treatment of diseases of the internal organs and it was called "acupuncture." This method involves the needle stick of individual "acupuncture points." In the current era on the basis of acupuncture has been added to action on the skin also low temperature, electromagnetic field, acoustic waves, laser rays, electric currents, irritants, etc. "Acupuncture" added more and more new points on the skin that correspond to individual organs of the inner. This is precisely the search for new sensitive acupuncture points on the skin of man, convinced by the Polish scientist Dr. Ferdinand Barbarianism for a brilliant application for "total acupuncture" or key therapy. In the latter method, the entire skin is gradually subjected to an impact while retaining a certain algorithm.

In the keypad, a "pre-reaction" treatment is performed on the scalp, which is the initial stage of diagnosis and/or therapy. The next stage is the stacking order of recovery using the entire body surface area. The treatments are performed with a clown (Łac. Clavus-Nail) on the surface of the skin, over the anatomy of the nervous system and in the zones and points of the biologically active including acupuncture. This procedure is provokes by stimulation of reflex neurophysiological processes-Dermowisceralne, to a group of receptors: Dermatome and further through the neurochemical signal relays are influenced by biocybernetic control on neuroses, litters, Sclerotome and via the nervous system can control the corrective action at the Endogenary-subcellular level. The described process of biocybernetics control allows extremely precise and complementary to regulate, through the nervous system, at endogenous level: cytokines, geniative, interferons, endorphins and other endogenous factors to which other methods Have no corrective access yet.

\section{Applications}

Key therapy is a modern diagnostic and therapeutic direction, extremely effective Read a Number of Important Scientific Publications Of The Author Of The Method Of The Keyboard in: Polish press, radio and television repeatedly were publications, reportages and movies, type in Google: Let's talk Barbarianism TV Ferdinand. Write in Google: www.instytutklawiterapii.com there in the RIGHT margin is YT logo 8 minutes video, WHERE the bureau ZG. The Polish Medical Society announces its opinion on monitoring the effectiveness of the keyboard method from 80 to $20 \mathrm{TH}$ century in the neurological clinic of the central Clinical Hospital of MSW in Warsaw. On this page, the e-mail is a notice of the new edition of the editor of the key book in 3 volumes with clinical evidence during the 8 hours, which take place Every first Saturday of the month in the conference center MAZOWSZE in Otrębusach At Świerkowej Street 2, where Dr. Ferdinand Barbarianism points out how to select biocybernetics algorithms for diagnosing doctors. After a lunch break, Dr. Ferdinand Barbarianism makes a publicly- based diagnosis of aponeurotic related to his clinical findings in the reading of neuropathy and polyne pathy and on the surface of the dermis of neurons with meta- Diagnosis of malignant tumors at the pathomorphological level. And also how to dispose of neuropathy and glilosis of neurons without medication in a few days. For more information on clinical evidence, see the English language in the following publications:

Weekly Polish voice ARTICLE: Klawiter Apia dated 11-18 May 2010 year (CANADA and USA).

Read the International Clinical Pathology Journal: ICPJL-02-00041 AND ICPJL-02-00042 (June 2016), AND ICPJL-160P-162, AND ICPJL-os-163 (July 2016); ICPJ 16-CON-170; ICPJL16-CON-171, molecular method in the Biocybernetics clavi therapy, noninvasive, Ferdinand Barbasiewicz, author.

Read the manuscript cancer in Current Trends in Biomedical, Engineering and Biosciences (CTBEB), Journal, USA, Ferdinand Barbasiewicz. 3d Biomaterials Journal. F. Barbasiewicz Integrative Clinical Medicine Journal, F. Barbasiewicz Prof. Kazuhisa Bessho University Kyoto Library Publish, Ferdinand Barbasiewicz, Canadian scientific Internet, publications by Ferdin and Barbasiewicz EC Proteomies and Bioinformatics, Ferdin and Barbasiewicz Typein Google: Clavitherapy. Microbiology Journal, microbiology and Infectious Diseases!!USA, Ferdyn and Barbasiewicz International Journal of Medical Science and Clinical invention ijmsci ISSN (Online): 2348-991x: (Print): 2454-9576, Ijmsci Index Copernicus Value for: (2016) 77.02 (?). - Ferdin and Barbasiewicz. Loj Medical Sciences (LOJMS) USA, Ferdin and Barbasieicz Journal of Pathology and Pathogenic Research, Barbasiewicz Ferdinand.

International Journal of Medical Research and Studies (Int. J. Med. Res. and Std.), Ferdinand Barbasiewicz.

Fortune journal, Ferdyn and Barbasiewicz. Peer Reviewed Journal of Forensic and Genetic Sciences (PRJFGS) ISSN 2638 -6062, Ferdyn and Barbasiewicz. We hereby inform you that we have published your article ANN-18-MRW-542 entitled "Molecular Method in the Biocybernetics Clavi therapy, Non Invasive, Neuropathic and Oncology" in Archives in Neurology and Neuroscience. Please acknowledge the following URL to access yourarticle https://irispublishers.com/ann/index.php Microbiology and Infectious Diseases! - Ferdinand Barbasiewicz.

\section{Volume 3 Issue 11 November 2019 (C) All rights are reserved by Patient.}

